\title{
Flexible chain molecules in the marginal and concentrated regimes: Universal static scaling laws and cross-over predictions
}

\author{
Manuel Laso ${ }^{\text {a) }}$ and Nikos Ch. Karayiannis \\ Institute for Optoelectronics and Microsystems (ISOM), UPM José Gutiérrez, Abascal 2, E-28006 Madrid, \\ Spain
}

(Received 21 February 2008; accepted 28 March 2008; published online 5 May 2008)

\begin{abstract}
We present predictions for the static scaling exponents and for the cross-over polymer volumetric fractions in the marginal and concentrated solution regimes. Corrections for finite chain length are made. Predictions are based on an analysis of correlated fluctuations in density and chain length, in a semigrand ensemble in which mers and solvent sites exchange identities. Cross-over volumetric fractions are found to be chain length independent to first order, although reciprocal- $N$ corrections are also estimated. Predicted scaling exponents and cross-over regimes are compared with available data from extensive off-lattice Monte Carlo simulations [Karayiannis and Laso, Phys. Rev. Lett. 100, 050602 (2008)] on freely jointed, hard-sphere chains of average lengths from $N=12-500$ and at packing densities from dilute ones up to the maximally random jammed state. () 2008 American Institute of Physics. [DOI: 10.1063/1.2912189]
\end{abstract}

\section{INTRODUCTION}

The pioneering concept of scaling laws that dictate the static and dynamic behaviors of macromolecular systems irrespective of their chemical constitution is widely considered as one of the most significant and fundamental advances in polymer science. ${ }^{1-3}$ This well established universal behavior allows for a systematic unification of experimental and simulation data by limiting the set of dominant mechanisms and corresponding parameters. Consequently, universal scaling laws greatly simplify the prediction of structure-property relations, an objective of paramount importance for the design of novel polymeric materials. From the theoretical point of view, it is often required to resort to simplified descriptions that deviate from realistic molecular models in order to achieve mathematical tractability. The freely jointed, hardsphere chain is perhaps the simplest of these idealizations which incorporates the fundamental concept of excluded volume.

While static and dynamic scaling laws (along with the corresponding crossover predictions) for dilute and semidilute polymer systems have been proposed theoretically and have been verified by a large number of experimental ${ }^{4-6}$ and modeling $^{8-12}$ studies, the marginal and concentrated regimes have been comparatively less well explored. These regimes are often introduced as those intervals of polymer volumetric fraction or concentration where the quadratic (marginal) or cubic (concentrated) terms in a series expansion of the chemical potential difference predicted by the FloryHuggins theory become dominant. ${ }^{13,14}$ Although both interesting in their own right, the marginal and concentrated regimes have not received the degree of attention commanded by the scaling and renormalization approaches valid in the dilute and semidilute regimes. Scaling laws in the marginal

\footnotetext{
${ }^{a)}$ Author to whom correspondence should be addressed. Electronic mail: mlaso@etsii.upm.es.
}

and concentrated regimes were first postulated by Edwards ${ }^{15}$ and partially confirmed by experiments. ${ }^{4,16}$ The marginal regime, in particular, was absent in the original formulation by the French school, and its very existence has even been the subject of some controversy. ${ }^{4,14}$ Schaefer proposed an alternative to the scaling law approach in the marginal regime. ${ }^{14}$ On the other hand, the exploration of the marginal and concentrated regimes presents considerable experimental challenges. ${ }^{17,18}$ It is remarkable that scaling laws for blob size in all regimes have been proposed but not always for chain size. ${ }^{13,14}$ In very recent works, Wittmer et al. and Beckrich et al. ${ }^{19,20}$ have reviewed and extended Flory's ideality hypothesis.

From the computational point of view, the marginal regime has been reached in only a few instances for off-lattice models, ${ }^{8,21-23}$ while the concentrated regime has remained inaccessible until very recently. ${ }^{24,25}$ Using a general Monte Carlo (MC) scheme ${ }^{25}$ we were able to generate long trajectories of model configurations of freely jointed chains of tangent hard spheres at packing densities from dilute ones up to the close vicinity of the maximally random jammed ${ }^{26-28}$ (MRJ) state $\left(\varphi=\varphi^{\mathrm{MRJ}}\right)$. By generating and successively equilibrating long hard-sphere chains (for average molecular lengths up to $N=500)$ in the whole density range $(0<\varphi$ $<\varphi^{\mathrm{MRJ} J}$ ) it is now possible to determine the static scaling regimes, from the dilute up to the concentrated, along with the corresponding characteristic cross-over packing densities.

Complementary to these simulations near the MRJ state we present predictions for the scaling exponents and the cross-over volumetric fractions in the marginal and concentrated solution regimes. We describe a general correspondence between polymer solution configurations and hardsphere packings, both at the MRJ state. This correspondence is the basis of a classification scheme employed to single out the microscopic mechanisms of correlated fluctuations in volumetric fraction and chain length. 


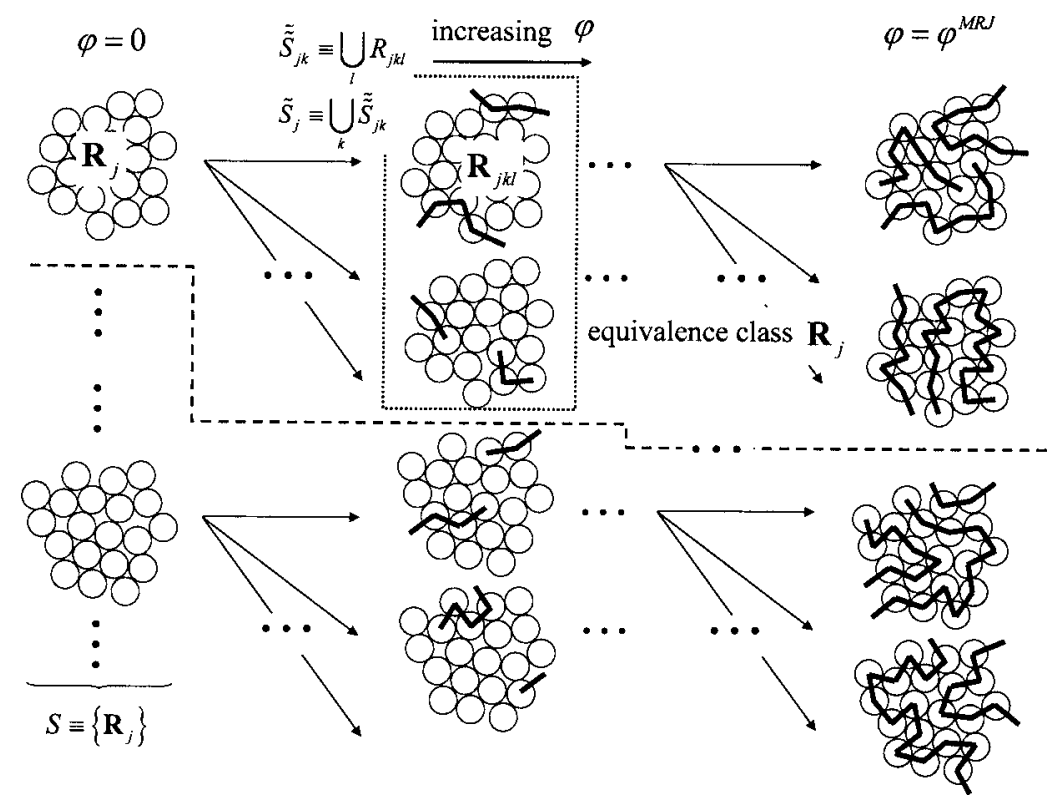

FIG. 1. Schematic representation of ensemble $\widetilde{\Xi}$. Polymer volumetric fraction increases from left $\varphi=0$ to right $\varphi=\varphi^{\text {MRJ. }}$. $\widetilde{\Xi}$ is split in equivalence classes represented by the single-sphere configuration $\mathbf{R}_{j}$. The dashed line marks the boundary of equivalence class $\mathbf{R}_{j}$. The dotted rectangle represents $\widetilde{\mathbf{R}}_{j}^{k}$, a set of configurations with prescribed chain length distribution, that share $\mathbf{R}_{j}$ but differ in the connectivity.

\section{HARD-SPHERE REPRESENTATION OF SOLUTIONS OF FLEXIBLE HOMOPOLYMERS}

Let $j$ be the label of the $j$ th configuration of a system of $N_{\text {sites }}$ identical hard spheres of unit volume, occupying a volume of $V$ units at the MRJ state. Let $\mathbf{R}_{j}$ be the list of the position vectors of the centers, or "sites," of its $N_{\text {sites }}$ spheres: $\mathbf{R}_{j} \equiv\left\{\cup_{i=1}^{N_{\text {sites }}} \mathbf{r}^{(i)}\right\}$. Let $S \equiv\left\{\mathbf{R}_{j}\right\}$ be the complete set, or ensemble, of configurations of $N_{\text {sites }}$ hard spheres at the MRJ state. Transformations of $\mathbf{R}_{j}$ by the translation, proper and improper orthogonal, and subindex permutation groups are excluded from $S$. Each $\mathbf{R}_{j}$ in $S$ then represents a physically unique member of this ensemble.

From $\varphi_{\text {chains }}^{\mathrm{MRJ}}=\varphi_{\text {spheres }}^{\mathrm{MRJ}}($ as shown in Ref. 24) and from the definition of the MRJ state it follows that there exists a surjective mapping between the set of hard-sphere chain ("polymer") configurations and the ensemble $S$ of single hard-sphere configurations at the MRJ state: For every member $\mathbf{R}_{j} \in S$, it is possible to exhaustively generate a large, but finite, set of configurations containing a total of $N_{\text {sites }}$, and having a given volumetric fraction, or "density," of chain molecules $\varphi$, defined as

$$
\varphi=\frac{\sum_{i=2}^{c} i N_{i}}{V}=\frac{\sum_{i=2}^{c} i N_{i}}{N_{\text {sites }}} \varphi^{\mathrm{MRJ}},
$$

where $c$ is the number of distinct chain lengths (species), and the index $i=1$ is assigned to single spheres ("solvent"). The volumetric fraction, or density, of the solvent is $\varphi_{\text {sol }}=N_{1} / V$ $=\varphi^{\mathrm{MRJ}}-\varphi$. Within this set it is possible to distinguish subsets of configurations containing a prescribed distribution of chain lengths, i.e., $N_{i}$ chains of length $i, i=1, \ldots, c$, such that Eq. (1) is satisfied. Such polydisperse chain configurations at the MRJ can be obtained from a given $\mathbf{R}_{j} \in S$ by tracing sets of $N_{\text {chains }}=\sum_{i=1}^{c} N_{i}$ nonoverlapping paths of the prescribed chain length distribution, that connect tangent hard spheres in the hard-sphere MRJ configuration $\mathbf{R}_{j}\left(N_{1}\right.$, the number of one-site "chains," or solvent sites, is included in the sums). Let $\mathbf{R}_{j k}$ denote (the $k$ th) set of all path configurations that can be obtained from $\mathbf{R}_{j}$ by specifying volume fraction $\varphi$ and (the $k$ th) chain length distribution, i.e., $k$ counts chain length distributions, but does not distinguish individual realizations. Let $\mathbf{R}_{j k l}$ denote (the $l$ th) set of all configurations that can be obtained from $\mathbf{R}_{j k}$ by the $l$ th path trace, i.e., obtained solely by altering the connectivity between sites, while maintaining volumetric fraction and chain length distribution. $l$ counts individual chains (paths or graphs) that connect sites with fixed coordinates $\mathbf{R}_{j}$ that have a prescribed chain length distribution at a given volumetric fraction.

The set $\tilde{\tilde{S}}_{j k} \equiv \cup_{l} \mathbf{R}_{j k l}$ is then the ensemble of configurations of chain molecules of prescribed length distribution, and volumetric fraction [Eq. (1)], and constrained by $\sum_{i=1}^{c} i N_{i}=N_{\text {sites }}$. Transformations of $\mathbf{R}_{j k l}$ by permutations under which the connectivity graph of the configuration $\mathbf{R}_{j k l}$ is invariant, i.e., chain relabeling, are excluded from $\tilde{\tilde{S}}$.

The set $\widetilde{S}_{j} \equiv \cup_{k} \tilde{\tilde{S}}_{j k}$ is the ensemble of configurations of chain molecules that can be obtained from $\mathbf{R}_{j}$, of prescribed volumetric fraction (1) and constrained by $\sum_{i=1}^{c} i N_{i}=N_{\text {sites }}$. Finally, let $\widetilde{\Xi}=\cup_{j} \widetilde{S}_{j}$ be the ensemble of all possible paths, that can be obtained from all $\mathbf{R}_{j}$, for all volumetric fractions and for all possible prescriptions of chain length distribution, with the only constraint that $\sum_{i=1}^{c} i N_{i}=N_{\text {sites. }}$. $\widetilde{\Xi}$ is, from the configurational point of view, an isochoric grand canonical ensemble in which the number of molecules of each species can fluctuate, subjected to the restriction $\sum_{i=1}^{c} i N_{i}=N_{\text {sites }}$. $\widetilde{\Xi}$ splits naturally in classes of equivalence by the relation $\sim$ defined by

$$
\mathbf{R}_{j k l} \sim \mathbf{R}_{m n p} \quad \text { iff } j=m
$$

(see Fig. 1). Note that $\mathbf{R}_{j}$ is also a suitable representative of the smaller set $\widetilde{\Xi} / \sim$ formed from $\widetilde{\Xi}$ by the action of $\sim$. Each class $\mathbf{R}_{j}$ comprises all physically distinct configurations that differ only in the way $N_{\text {sites }}$ sites of fixed coordinates are connected. The surjection between $\tilde{\Xi}$ and $S$ is the mapping between any $\mathbf{R}_{j k l} \in \widetilde{\Xi}$ and the class representative $\mathbf{R}_{j}$ obtained by deletion of connectivity of $\mathbf{R}_{j k l} \in \widetilde{\Xi}$. For large 
values of $N_{\text {sites }}$, i.e., in the macroscopic limit, the class $\mathbf{R}_{j}$ is properly parametrized by the volumetric fraction $\varphi$.

Thus, given the observed equality of $\varphi_{\text {chains }}^{\mathrm{MRJ}}=\varphi_{\text {spheres }}^{\mathrm{MRJ}}{ }^{24}$ the ensemble $\widetilde{\Xi}$ comprises all microscopic instances of polydisperse solutions of athermal chain molecules in an athermal solvent at all densities in the range $\left[0, \varphi^{\mathrm{MRJ}}\right]$. In view of the very small compressibility of liquid polymer solutions, $\widetilde{\Xi}$ is an adequate setting for the discussion of universal scaling behavior of polymers in solution. ${ }^{29}$ As will be shown below, the simplicity of the intermolecular potential makes it possible to predict static scaling exponents and cross-over concentrations in the marginal and concentrated regimes based on statistical mechanical, microscopic arguments.

The surjection between $\widetilde{\Xi}$ and $S$ allows the investigation of the effect of polymer concentration (density) by continuously varying $\varphi$, while $\varphi^{\mathrm{MRJ}}=\varphi+\varphi_{\text {sol }}$ remains constant. In the very dilute regime $N_{1}=N_{\text {sites }}$, whereas $N_{1}=0$ in the melt. We base our analysis on a graph-theoretical proof that static scaling behavior can be ascertained by monitoring the microscopic mechanisms of strictly local correlated fluctuations of volumetric fraction $\varphi$ and chain length in a semigrand canonical ensemble. These mechanisms are especially transparent for the hard-sphere pair-wise potential at $\varphi+\varphi_{\text {sol }}=\varphi^{\mathrm{MRJ}}$ since scaling and cross-over are based on straightforward combinatorial/graph connectivity arguments.

The basis of semigrand ensembles ${ }^{30,31}$ is a resummation of the grand canonical partition function in terms of molecular species identities. As a starting point we take the grand canonical partition function $\Xi$ for a multicomponent mixture containing $c$ components:

$$
\begin{aligned}
\Xi\left(V, T, \mu_{1}, \ldots, \mu_{c}\right)= & \sum_{N_{1}, \ldots, N_{c}}^{\infty} \prod_{i=1}^{c} \frac{q_{i}^{N_{i}} \exp \left(\beta \mu_{i} N_{i}\right)}{N_{i} !} V^{N} \\
& \times \int d^{3 N} \mathbf{R} \exp (-\beta U(\mathbf{R})),
\end{aligned}
$$

with $N=\sum_{i=1}^{c} N_{c}$, and $q_{i}$ are the translational and internal contributions to the partition function for species $i$, and

$$
Z\left(V, T, N_{1}, \ldots, N_{c}\right)=\int d^{3 N} \mathbf{R} \exp (-\beta U(\mathbf{R}))
$$

is the classical configurational integral. Imposing a constraint of fixed total number of molecules $N$, eliminating $N_{1}$, and multiplying both sides of the sum [Eq. (3)] by $\exp \left(-\beta \mu_{1} N\right)$, we obtain

$$
\begin{aligned}
& \Xi\left(V, T, \mu_{1}, \ldots, \mu_{c}\right) \exp \left(-\beta \mu_{1} N\right) \\
& =\sum_{N_{2}, \ldots, N_{c}}^{\infty}{ }^{\prime} q_{1}^{N} \prod_{i=1}^{c}\left(\frac{q_{i}}{q_{1}}\right)^{N_{i}} \frac{\exp \left[\beta\left(\mu_{i}-\mu_{1}\right) N_{i}\right]}{N_{i} !} V^{N} \\
& \quad \times \int d^{3 N} \mathbf{R} \exp (-\beta U(\mathbf{R})) .
\end{aligned}
$$

The primed sum $\left(\Sigma^{\prime}\right)$ implies constrained summation keeping $N$ constant. The sum over the number of molecules of each species [Eq. (4)] can now be rewritten as a sum over the identities of molecules, including the correction factor for multiple counting,

$$
\begin{aligned}
Y= & \sum_{\text {identities }}^{\infty}, \frac{q_{1}^{N}}{N !} \prod_{i=1}^{c}\left(\frac{q_{i}}{q_{1}}\right)^{N_{i}} \\
& \times \exp \left[\beta\left(\mu_{i}-\mu_{1}\right) N_{i}\right] V^{N} Z\left(V, T, N_{1}, \ldots, N_{c}\right) .
\end{aligned}
$$

Expression (5) constitutes the basis of a number of MC schemes based on the strategy of allowing species to change identity. In its original form the semigrand formalism was used to study polydisperse mixtures of spherical (single site) components, ${ }^{31,32}$ but it has also been applied with great success to chain molecular systems, ${ }^{33-40}$ often in combination with sophisticated chain connectivity-altering $\mathrm{MC}$ schemes ${ }^{33,41,42}$ such as the end bridging, double bridging, and intramolecular double rebridging moves in which chain molecules swap identities by exchanging multisegment fractions. This latter type of simulations is based on an extension of the original semigrand ensemble in which a second constraint is added: Not only must the number of chain monomers remain constant, but the total number of chain molecules is fixed as well, $\sum_{i=2}^{c} i N_{i}=N_{\text {sites }}, \sum_{i=2}^{c} N_{i}=N_{\text {chains }}$ using the present nomenclature. While ideally suited for connectivity-altering schemes, the doubly constrained semigrand ensemble does not allow an investigation of polymer volumetric fraction effects, even in its isobaric variant.

Hence, we have chosen a singly constrained $\left(\sum_{i=1}^{c} i N_{i}\right.$ $=N_{\text {sites }}$ ) ensemble which is more suitable to include the additional degree of freedom represented by variable polymer/ solvent volumetric fraction. We have also taken the resummation strategy [Eq. (5)] one step further by considering identity exchanges between individual mers of the chain molecules for all chain lengths. In particular, identity exchanges involving $i=1$ correspond to solvent particles being transformed into chain mers and vice versa. Thus, fluctuations in $N_{1}$ are directly correlated with fluctuations in $\varphi$ and completely decorrelated with fluctuations in the remaining $N_{i}$,

$i=2, \ldots, c$,

$$
\delta \varphi=-\delta\left(\frac{N_{1}}{N_{\text {sites }}}\right)=-\frac{\delta N_{1}}{N_{\text {sites }}},
$$

by virtue of the constraint $\sum_{i=1}^{c} i N_{i}=N_{\text {sites }}$.

A final condition is needed in order for ensemble configurations to be representative of polymer solutions: A clear separation between the molecular weight of the solvent $(i=1)$ and the shortest chains $\left(i=l_{\min } \gg 1\right)$. This separation will be achieved by specifying a suitable spectrum of chemical potentials. Imposition of chemical potentials entails a Legendre transformation of the ensemble. The $\left[V T N_{\text {sites }} \mu^{*}\right]$ ensemble is thus obtained by arbitrarily choosing a single species as reference species. The solvent $(i=1)$ will be chosen for convenience. $N_{i}$ is eliminated by means of the constraint $\sum_{i=1}^{c} i N_{i}=N_{\text {sites}}$, and the differential free energy is Legendre transformed with respect to all species numbers $N_{i}$ except the reference, so that the semigrand $\left[V T N_{\text {sites }} \mu^{*}\right]$ thermodynamic potential is obtained,

$$
\Upsilon=A-\sum_{i=2}^{c} \mu_{i}^{*} N_{i}
$$




$$
d \Upsilon=-S d T-P d V+\sum_{i=2}^{c} N_{i} d \mu_{i}^{*}+\mu^{\left(N_{\text {sites }}\right)} d N_{\text {sites }},
$$

where

$$
\mu_{i}^{*}=\mu_{i}-i \mu_{1} \quad \mu_{i}^{\left(N_{\text {sites }}\right)}=\mu_{1} .
$$

The link to the microscopic level is

$$
\mathrm{Y}=-k_{B} T \ln \Upsilon^{*},
$$

with the following partition function:

$$
\begin{aligned}
\Upsilon^{*}\left[V, T, N_{\text {sites }}, \mu_{i}^{*}\right] & \\
= & \sum_{\text {site identities }}^{\infty}, \frac{q_{1}^{N}}{N !} \prod_{i=1}^{c}\left(\frac{q_{i}}{q_{1}}\right)^{N_{i}} \exp \left[\beta \mu_{i}^{*} N_{i}\right] V^{N} \\
& \times \frac{1}{N_{1} ! N_{2} ! \cdots N_{c} !}\left(\frac{2 \pi m k_{B} T}{h^{2}}\right)^{3 N_{\text {sites }}} Z\left(V, T, N_{1}, \ldots, N_{c}\right) .
\end{aligned}
$$

The constrained summation is performed over configurations $\mathbf{R} \in \widetilde{\Xi}$, which satisfy $\sum_{i=1}^{c} i N_{i}=N_{\text {sites }}$.

In this isochoric, semigrand canonical ensemble $\left[V T N_{\text {sites }} \mu^{*}\right]$, total volume $V$, temperature $T$, total number of sites $N_{\text {sites}}$, and a spectrum of chemical potentials are specified. Conjugate variables $\left[P, U, N_{i}, \mu_{i}^{\left(N_{\text {sites }}\right)}=\mu_{I}\right]$ fluctuate. In particular, the number of solvent sites and hence [Eq. (6)] polymer volumetric fraction can fluctuate. The average polymer length distribution results from the imposed spectrum of chemical potentials. The chemical potential of the reference species is computed a posteriori, e.g., via a suitable semigrand modification ${ }^{31}$ of Widom's insertion method. ${ }^{43}$

A simple length scale separation between solvent and polymer can be achieved by specifying

$$
\mu_{i}^{*}=\left\{\begin{array}{cc}
0 & \text { for } i=1, \text { or } i \geqslant l_{\min } \\
\infty & \text { for } 1<i<l_{\min }
\end{array}\right.
$$

Although the resulting distribution of chain lengths cannot be computed exactly, it cannot differ much from the imposed set of activities. ${ }^{32}$ Alternatively, a maximum term analysis ${ }^{44}$ can be used to determine an analytical approximation. ${ }^{33}$ This is not required for our purposes, since Eq. (12) ensures that short chains are suppressed, while the numbers of solvent and chain molecules can fluctuate. ${ }^{45}$

Fluctuations in polymer volumetric fraction are related to fluctuations in the number of solvent sites via Eq. (6) and through

$$
\left\langle\left(\delta N_{1}\right)^{2}\right\rangle=\left(\frac{\partial\left\langle N_{1}\right\rangle}{\partial \beta \mu_{1}}\right)_{\beta, V} .
$$

In general, Eq. (13) cannot be evaluated analytically. However, in the marginal and concentrated regimes, it will be shown that an analysis of the distribution of states $\mathbf{R} \in \widetilde{\Xi}$ in connectivity space yields valuable insights. Equation (11) associates to each of the states $\mathbf{R} \in \widetilde{\Xi}$ a probability proportional to

$$
\exp \left[\sum_{i=l_{\min }}^{c} \beta \mu_{i}^{*} N_{i}-\beta U(\mathbf{R})\right]
$$

where $U(\mathbf{R})=0, \forall \mathbf{R} \in \widetilde{\Xi}$. Also, since $l_{\text {min }} \gg 1$, the average "mer" or incremental chemical potential $\mu^{\text {mer }}$ $\equiv \sum_{i=2}^{c}\left(N_{i}\left\langle\mu_{i}\right\rangle / i\right) /(c-1)$ will, to excellent approximation, satisfy

$$
\mu^{\mathrm{mer}}=\mu_{1}
$$

Equation (15) is known to hold accurately for chemically realistic homopolymers. ${ }^{46}$ Thus, Eq. (14) trivially evaluates to $\exp \left[\sum_{i=l_{\min }^{c}} \beta \mu_{i}^{*} N_{i}-\beta U(\mathbf{R})\right]=1, \forall \mathbf{R} \in \widetilde{\Xi}$. All $\mathbf{R} \in \widetilde{\Xi}$ are equally probable. This result emphasizes the fact that the entire complication of evaluating the partition function and of sampling with probability density [Eq. (14)] are of a combinatorial (connectivity) nature.

\section{POLYMER SOLUTIONS IN THE MARGINAL AND CONCENTRATED REGIMES}

Regarding chain dimensions, the scaling theory for polymers as pioneered by de Gennes and co-workers ${ }^{2,4-6}$ predicts exponents for blob size dependence on $N$ (see note in Ref. 7) and $\varphi$, which are regime specific. In some cases blob scaling laws can be converted into scaling laws for radius of gyration. ${ }^{13,14}$ In particular, in the dilute regime, $\varphi \leqslant \varphi^{* \text {,semi }}$, where the cross-over packing fraction is $\varphi^{*, \mathrm{semi}}=N^{1-3 \nu}$, chains behave as self-avoiding random walks with $\left\langle R^{2}\right\rangle$ $\sim N^{2 \nu}$ where the Flory exponent $\nu=0.588$ is accurately known from renormalization group calculations. ${ }^{47}$ In the semidilute regime $\left(\varphi \geqslant \varphi^{* \text {,semi }}\right)$ a cross-over behavior is expected, together with a gradual decrease in the Flory exponent down to $\nu=0.5$, a characteristic of dense polymer melts. The scaling theory also predicts that in the semidilute regime chain dimensions (squared end-to-end distance) exhibit a density dependence of the form $\left\langle R^{2}\right\rangle \propto N \varphi^{\gamma}$ where $\gamma=(1-2 \nu) /(3 \nu-1) \simeq-0.230$.

The semigrand ensemble introduced above allows a direct examination of the universal features of the dependence of chain size, e.g., $\left\langle R^{2}\right\rangle$ or its characteristic ratio $C_{N}=\left\langle R^{2}\right\rangle / N b^{2}$, where $b$ is the bond length, on polymer volumetric fraction. A convenient device is to consider the effect of the fluctuations in $\varphi$ as the system visits ensemble states, i.e., fluctuations caused by transitions between individual members $\mathbf{R}_{j k l}$ and $\mathbf{R}_{m n p}$ of the ensemble $\widetilde{\Xi}$. These transitions obviously do not correspond to the actual dynamics of the system. Such unphysical transitions are frequently at the heart of efficient MC schemes, and they can be specified in any convenient way that respects proper sampling from $\widetilde{\Xi}$. In the following it is assumed that the system is ergodic (or in terms of a MC simulation that proper configurational sampling is guaranteed).

Irrespective of volumetric fraction, transitions $\mathbf{R}_{j k l}$ $\rightarrow \mathbf{R}_{\text {mnp }}$ are naturally classified in two disjoint, exhaustive types as follows: 


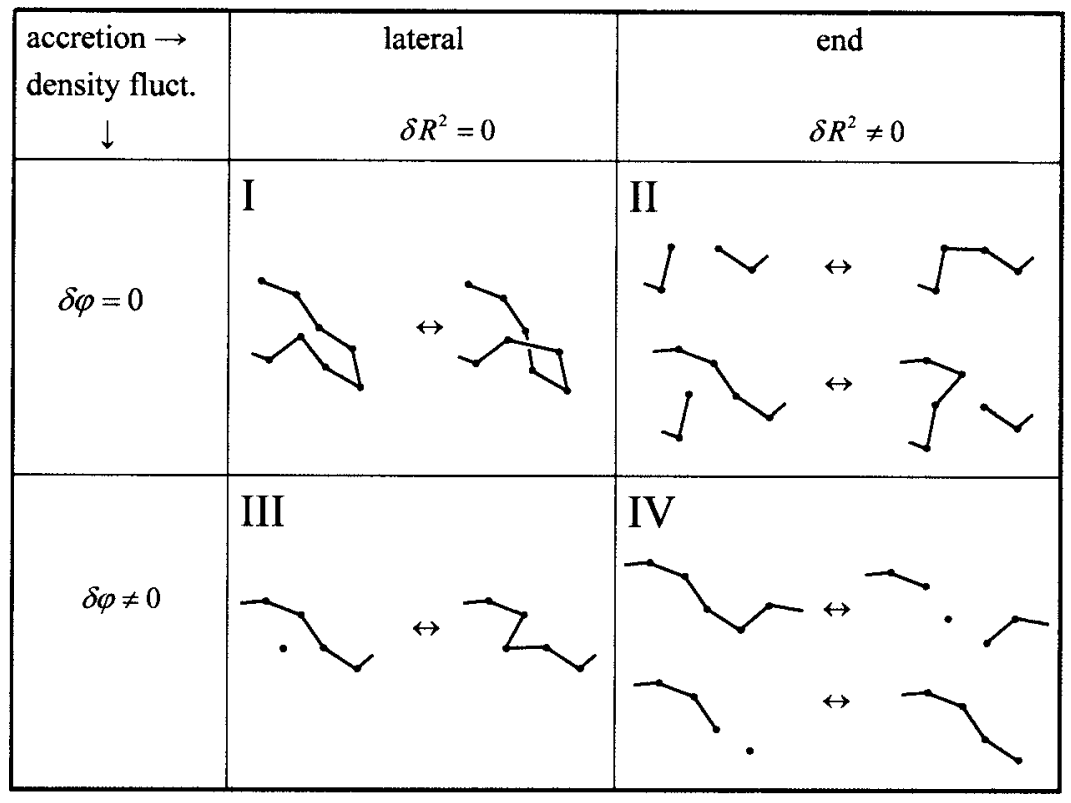

FIG. 2. Connectivity reconstruction moves, classified according to the type of chain growth/shrinkage ("accretion"), and to the volumetric fraction fluctuation they cause. Selected sample transitions are shown.

$$
\begin{aligned}
& \text { (A) } \quad \mathbf{R}_{j k l} \rightarrow \mathbf{R}_{m n p}, \quad j \neq m, \\
& \text { (B) } \mathbf{R}_{j k l} \rightarrow \mathbf{R}_{m n p} ; \quad j=m .
\end{aligned}
$$

In (A) the system visits configurations that belong to separate equivalence classes $\mathbf{R}_{j}$ and $\mathbf{R}_{m}$, while in (B) transitions take place between configurations that belong to the same class $\mathbf{R}_{j}$. In Fig. 1, (A) corresponds to transitions that cross the dashed line separating equivalence class $\mathbf{R}_{j}$ from other classes $\mathbf{R}_{k}$. Type (B) transitions correspond to a general change in connectivity within class $\mathbf{R}_{j}$ with the only constraint that $N_{\text {sites }}$ remains constant. Polymer volumetric fraction may change both in (A) and in (B) transitions. The definition of the MRJ state for single spheres, in particular, its maximal randomness, guarantees equal statistical significance of all configurations in set $S \equiv\left\{\mathbf{R}_{j}\right\}$ (at zero polymer volumetric fraction) and of all the represented equivalence classes $\mathbf{R}_{j}$. Hence, it suffices to consider type (B) transitions within class $\mathbf{R}_{j}$. A system transition within equivalence class $\mathbf{R}_{j}$ is tantamount to a partial or total reconstruction of the connectivity among sites whose fixed coordinates are specified by the list $\mathbf{R}_{i}$. Reduction to individual class $\mathbf{R}_{j}$ converts the continuous configurational problem into a discrete connectivity problem. Any such connectivity reconstruction is decomposable in a finite number of simpler moves, ${ }^{48}$ which for our purpose can be organized into four categories, which are shown in Fig. 2 together with some representative graphs. Two criteria are used in this classification: Whether or not the transition involves a volumetric fraction fluctuation, and whether or not the transition causes a change in the end-to-end distance of the chains involved. An ergodic trajectory in connectivity space, in which the system visits all states in $\widetilde{\Xi}$, can be constructed by specifying a microscopically reversible sequence of transitions, all of which necessarily fall into one of the four categories shown in Fig. 2. The effect of such transitions is to sample polydispersity and so to ensure equilibration of the chain length distribution, while allowing volumetric fraction $\varphi$ and $N_{i}$ to fluctuate. The inter- play between the allowed number of states and the effect of transitions on the conformation and size of the chains involved in the transition, etc., will result in the macroscopic observable (e.g., $\left\langle R^{2}\right\rangle$ ) characteristic of each regime. Predicting the outcome of this interplay is in general impossible, since it is of a difficulty comparable to the evaluation of averages over Eq. (11). However, at high polymer volume fractions partially analytical results will be obtained based on graph theoretic arguments to be developed in the next section.

\section{CONCENTRATED REGIME}

As a convenient starting point to obtain the universal static scaling behavior close to the MRJ we consider the system in the vicinity of the $\operatorname{MRJ}: \mathbf{R}_{j}(\varphi), \varphi \simeq \varphi^{\mathrm{MRJ}}$, and monitor fluctuations $\varphi=\varphi^{\mathrm{MRJ}}-\delta \varphi$ from the MRJ state that involve mutation of a chain site into a solvent site (and vice versa). The system stays in the same $\mathbf{R}_{j}$ class under such transformations, but visits a state belonging to a different chain size distribution, so that $\mathbf{R}_{j k l} \rightarrow \mathbf{R}_{j n p}, k \neq n$. Furthermore, the final state, $\mathbf{R}_{j n p}$ is in general also reachable from other members of $\tilde{\tilde{S}}_{j k}$, so fluctuations in $\delta \varphi$ induce mixing ${ }^{48}$ in connectivity space. ${ }^{48}$ If we consider the set of all individual configurations $\mathbf{R}_{j n p}$ as a set of points or vertices $V=\left\{\mathbf{R}_{j n p}\right\}=\{i ; i=1, \cdots\}$ in state connectivity space, transitions $\mathbf{R}_{j k l} \leftrightarrow \mathbf{R}_{j n p}, k \neq n$, can be represented as a set of edges $E=\{e(i, j) ; i, j=1, \cdots\}$ joining two such states, so that mutual state accessibility is univocally mappable onto a nondirected state connectivity graph $G=(V, E){ }^{49}$ It is important to emphasize that vertices in the state connectivity graph represent individual configurations of the entire system $\mathbf{R}_{j n p}$. A bond between two vertices indicates that the transition $\mathbf{R}_{j k l} \leftrightarrow \mathbf{R}_{j n p}$, $k \neq n$, between them is possible by a site mutation. $G=(V, E)$ represents connectivity in a very high dimensional state connectivity space. It is not the connectivity graph of individual sites in a given configuration $\mathbf{R}_{\text {jnp }}$, i.e., it does not describe how sites are connected to form chains. 

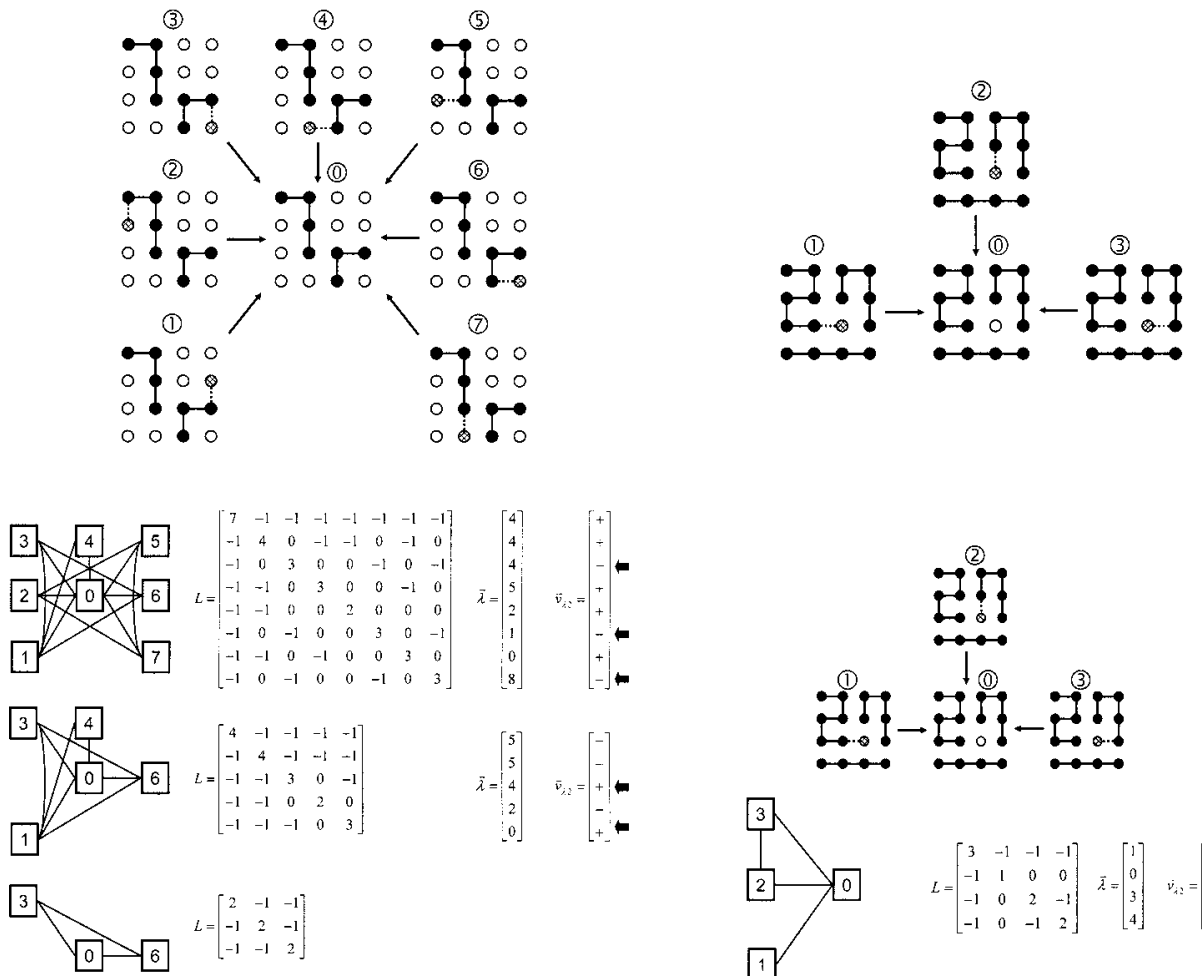

Graph $G$ contains information relevant for our fluctuation analysis, in particular, the degree of mixing ${ }^{51}$ of Markov chains on $G$. Our first goal is to obtain bounds for the degree of mixing in the neighborhood of the MRJ state (concentrated), $\varphi \simeq \varphi^{\mathrm{MRJ}}$, and far away from it (semidilute). As a first step in quantifying the degree of mixing, it is useful to consider the Laplacian matrix $L(G)$ of the connectivity graph. The Laplacian matrix of a graph $G$ containing $n$ vertices is an $n \times n$ symmetric matrix whose elements are defined as follows:

$$
L(G)_{i j}= \begin{cases}\# \text { of bonds ending in the vertex, } & \text { if } i=j \\ 0 & \text { if } i \neq j \text { and vertices } i \text { and } j \text { are not connected } \\ -1 & \text { if } i \neq j \text { and vertices } i \text { and } j \text { are connected. }\end{cases}
$$

The diagonal entry $L(G)_{i i}$ is the degree of vertex $i$ and counts the number of vertices that can be reached directly from vertex $i$. Figure 3 presents a highly simplified example: A $4 \times 4$ grid on which a given state $\mathbf{R}_{j n p}$, labeled $\odot$, is surrounded by all states, labeled (1), (2),..., from which it can be reached, i.e., $\mathbf{R}_{j k l} \rightarrow \mathbf{R}_{j n p}, k \neq n$, by mutation of a single chain site into a solvent site. The associated graph and its Laplacian matrix $L$ are shown to its right, both for an idealized semidilute (top) and for a concentrated (bottom) solution. A useful property of $L(G)$ is that a partition of $G$ into connected graphs can be performed by computing the eigenvector (or Fiedler vector ${ }^{50}$ ) $\vec{\nu}_{\lambda 2}$ associated with $\lambda_{2}$, and assigning nodes for which $\vec{\nu}_{\lambda 2_{i}}>0$ to one partition and nodes for which $\vec{\nu}_{\lambda 2_{i}} \leqslant 0$ to a second partition. Spectral partitioning can be recursively carried out to greater depths by discarding one of the partitions and repeating the process. Highly connected systems have graphs which can be partitioned deeply, and in which Markov chains mix rapidly (see below). This is shown explicitly in Fig. 3 for the graphs corresponding to the semidilute and the concentrated cases. Arrows pointing to elements in $\vec{\nu}_{\lambda 2}$ indicate the partition that is discarded at each step. Notice how in the concentrated case the partitioning scheme correctly groups configurations belonging to the same chain length distribution, i.e., members of the same $\tilde{\tilde{S}}_{j k}$. The very different degrees of connectivity of semidilute and concentrated solutions are also obvious even on a tiny grid.

We next recall key characteristics of a Markov chain of states (configurations) visited by the system in an ergodic path through connectivity space as follows:

- It is time homogeneous, has a finite state space $\mathcal{R} \subseteq \widetilde{\Xi}$, and has a transition matrix $P=\left(p_{i j}\right)_{i, j \in \mathcal{R}}$.

- Its stationary distribution $\pi=\left\{\pi_{i} ; i \in \mathcal{R}\right\}$ is uniform.

- Microscopic balance holds: $\pi_{i} p_{i j}=\pi_{j} p_{j i}, \forall i, j \in \mathcal{R}$. 
We formally define the underlying weighted graph associated with such a reversible Markov chain of states by assigning the set of vertices $V$ of the graph to the states of the system $\mathbf{R}_{j n p}$, and edges $e(i, j), i, j \in \mathcal{R}$, whenever the transition $i \leftrightarrow j$ is allowed. Edge $e(i, j)$ has weight $w_{i j}=\pi_{i} p_{i j}$ $=\pi_{j} p_{j i}$. We now make use of the fact that a finite ergodic Markov chain is "rapidly mixing" if and only if the conductance $^{51}$ of its underlying graph is large. The conductance of graph $G$ is defined as

$$
\Phi(G)=\min \left(\frac{\sum_{j \in V-C}^{i \in C} w_{i j}}{\sum_{i \in C} \pi_{i}}\right),
$$

where $C$ is a cut of the vertex set $V, C \subset V$, and the minimization is over all cuts. The rate of mixing is a measure of the deviation from the limiting distribution after $t$ steps of the Markov chain. A robust measure for this deviation from the limiting distribution is the relative pointwise distance from the limiting distribution $d(t)$,

$$
d(t)=\max \left\{\frac{p_{i j}(t)-\pi_{j}}{\pi_{j}}\right\} .
$$

By relating the conductance to the second eigenvalue of the transition matrix, it can be shown ${ }^{52}$ that the relative pointwise distance $d(t)$ to the stationary distribution is bounded by

$$
\left(1-\frac{\Phi(G)^{2}}{2}\right)^{t} \geqslant d(t)
$$

and rapid mixing results if a suitable lower bound on the conductance of the underlying graph $G$ can be established. In order to determine this bound, we have exploited the exact surjection between $\widetilde{\Xi}$ and $S$ for the hard-sphere chain/solvent systems to enumerate accessible neighbors in connectivity space for specific configurations at the MRJ. We used the min-load algorithm ${ }^{53}$ to find and enumerate a large number of sets of accessible neighbors for sample state connectivity graphs $G$ in the concentrated and in the semidilute regimes taken from Refs. 24 and 25. Exhaustive ensemble enumeration was not feasible, since it is an intractable \#NP-hard problem for the size of the available MRJ configurations, for which $N_{\text {sites }}$ is of the order of several thousands. It was however possible to numerically determine the following scalings for the ratio of the ensemble-average vertex degrees $L(G)_{i i}$ :

$$
\begin{aligned}
& \left\langle L(G)_{i i}\right\rangle_{\text {conc }}=O\left(\left\langle n_{\text {coord }}\right\rangle\right), \\
& \frac{\left\langle L(G)_{i i}\right\rangle_{\text {semi }}}{\left\langle L(G)_{i i}\right\rangle_{\text {conc }}} \propto\left(\frac{\varphi_{\text {sol }}^{\text {semi }}}{\varphi_{\text {sol }}^{\text {conc }}}\right)^{N_{\text {sites }},},
\end{aligned}
$$

where $\left\langle n_{\text {coord }}\right\rangle$ is the ensemble average coordination number. Results (20) and (21) are remarkable on two accounts: Not only is the vertex degree system-size independent in the concentrated regime [Eq. (20)], but it is also comparable to the coordination number in physical space. Equation (21) shows on the other hand the dramatic disparity in connectivities of the underlying graphs in the concentrated and semidilute re- gimes. Equations (20) and (21) are the desired result, since Eq. (21) is the condition that $G$ is dense ${ }^{49,52}$ in the semidilute regime, while it is not dense in the concentrated regime. In the dense case a lower bound for the graph conductance is given directly by

$$
\Phi(G) \geqslant \frac{1}{12 N_{\text {sites }}^{6}},
$$

(Theorem 3 of Jerrum and Sinclair ${ }^{52}$ ) and rapid mixing is assured.

Thus, as system size increases, the difference in state accessibility for the semidilute and concentrated regimes diverges exponentially fast. Individual system configurations in the concentrated regime have a limited number of directly accessible neighbors ("weak mixing"), whereas at lower volumetric fractions, states mix rapidly.

Rapid mixing is in this context equivalent to intractability, since an exponentially large number of graphs need to be evaluated to ascertain the effect of a fluctuation $\delta \varphi$ caused by site mutation. However, for a fluctuation in the concentrated regime, $\varphi \simeq \varphi^{\mathrm{MRJ}}$, solvent $\leftrightarrow$ mer mutations take place in a virtually pure chain environment, and the number of states (members of $\tilde{\tilde{S}}_{j k}$ ) from which a given mutated state $\mathbf{R}_{j k l}$ $\rightarrow \mathbf{R}_{j n p}, k \neq n$, is reachable is a small, constant value $O\left(n_{\text {coord }}\right) \delta \varphi$. This is in stark contrast to the general situation at $\varphi$ far from $\varphi^{\mathrm{MRJ}}$, where a given mutated state $\mathbf{R}_{j n p}$ can be reached from an exponentially large number of states. The desired final conclusion is that as $\varphi \rightarrow \varphi^{\mathrm{MRJ}}$ purely local perturbations in the set $\tilde{\tilde{S}}_{j k}$ are strictly valid, ${ }^{52}$ i.e., correlated fluctuations in chain dimensions and density can be ascertained from the effect of the fluctuation in the immediate physical-space vicinity of the mutated site.

Returning now to Fig. 2, transitions not involving a volumetric fraction fluctuation (first row, types I and II) remain in the constant density subset $\mathbf{R}_{j}(\varphi)$ (represented by the dotted line in Fig. 1). Consequently, no information regarding the $\left\langle R^{2}\right\rangle$ vs $\varphi$ scaling can be gleaned from them. Transitions involving a density fluctuation $\delta \varphi \neq 0$ (types III and IV, second row in Fig. 2) are thus key to scaling analysis. They will, in the general case, be analytically intractable.

However, in the specific case of high concentration and weak mixing in connectivity space, we can proceed as follows: First, chain size will scale as $\left\langle R^{2}\right\rangle \propto N$ due to screening, and the ratio $\left\langle R^{2}\right\rangle /\left\langle R_{g}^{2}\right\rangle$ of (squared) end-to-end distance to (squared) radius of gyration will be constant. Hence, scaling arguments for $\left\langle R^{2}\right\rangle$ will automatically be valid for $\left\langle R_{g}^{2}\right\rangle$, and vice versa. Furthermore, among the $\delta \varphi \neq 0$ transitions, type IV mutations, which we call "end accretion," will change both contour length, which is proportional to $N$ and $\left\langle R^{2}\right\rangle$, whereas type III transitions, "lateral accretion" mutations, will change the contour length $N$ of the chain while keeping its end-to-end distance $R^{2}$ strictly constant (configurationwise, not ensemble average). There is no contradiction in this with the (ensemble average) constancy of $\left\langle R^{2}\right\rangle /\left\langle R_{g}^{2}\right\rangle$ since lateral accretion is equally probable along the entire contour length, and the spatial distribution of the accreted site is the same as that of the existing chain mers, so that the radius of gyration for the chain after accretion $(N+1)$ sites is 


$$
\begin{aligned}
\left\langle R_{g}^{2}\right\rangle_{N+1}= & \frac{1}{(N+1)^{2}} \sum_{1 \leqslant i<j \leqslant N+1}\left\langle r_{i j}^{2}\right\rangle=\frac{1}{(N+1)^{2}}\left[\sum_{1 \leqslant i<j \leqslant N}\left\langle r_{i j}^{2}\right\rangle+\sum_{1 \leqslant i \leqslant N}\left\langle r_{i N+1}^{2}\right\rangle\right] \\
= & \frac{N^{2}}{(N+1)^{2}}\left\langle R_{g}^{2}\right\rangle_{N}+\frac{1}{(N+1)^{2}}\left\langle\sum_{1 \leqslant i \leqslant N}\left[\left(\mathbf{r}_{i}-\mathbf{r}_{\text {c.o.m. } . N}\right)+\left(\mathbf{r}_{\text {c.o.m. } . N}-\mathbf{r}_{N+1}\right)\right]^{2}\right\rangle \\
= & \frac{N^{2}}{(N+1)^{2}}\left\langle R_{g}^{2}\right\rangle_{N}+\frac{1}{(N+1)^{2}}\left[\sum_{1 \leqslant i \leqslant N}\left\langle\left(\mathbf{r}_{i}-\mathbf{r}_{\text {c.o.m. } . N}\right)^{2}\right\rangle+\sum_{1 \leqslant i \leqslant N}\left\langle\left(\mathbf{r}_{\text {c.o.m. } . N}-\mathbf{r}_{N+1}\right)^{2}\right\rangle\right. \\
& \left.+2 \sum_{1 \leqslant i \leqslant N}\left\langle\left(\mathbf{r}_{i}-\mathbf{r}_{\text {c.o.m. } . N}\right) \cdot\left(\mathbf{r}_{\text {c.o.m. } . N}-\mathbf{r}_{N+1}\right)\right\rangle\right]=\frac{N^{2}+2 N}{(N+1)^{2}}\left\langle R_{g}^{2}\right\rangle_{N}=\left\langle R_{g}^{2}\right\rangle_{N}+O\left(1 / N^{2}\right) .
\end{aligned}
$$

The remaining steps are now very straightforward: Taking into account microscopic reversibility, the probabilities of both lateral (type III) and end (type IV) transitions are

$$
\text { Type III: } O(1) \text { Type IV: } O(1)+O\left(N^{-1}\right) \text {, }
$$

the $O\left(N^{-1}\right)$ term stemming from chain-end mutation. However, the effects of both types of transitions on $R^{2}$ are quite different,

$$
\begin{aligned}
& \text { Type III: } l_{i} \rightarrow l_{i-1}+1, \quad \delta R^{2} \propto 0 \Rightarrow \frac{\left\langle R^{2}\right\rangle}{N} \propto \varphi^{-1}, \\
& \text { Type IV: } l_{i} \rightarrow l_{j}+l_{i-j-1}+1, \quad \delta \varphi=-\frac{1}{N_{\text {sites }}}, \\
& \delta R^{2} \propto \delta N \propto-\delta \varphi \Rightarrow \frac{\left\langle R^{2}\right\rangle}{N} \propto \varphi^{0} .
\end{aligned}
$$

Hence, to first order, the overall scaling behavior in the concentrated regime must be

$$
C_{N}=\frac{\left\langle R^{2}\right\rangle}{N b^{2}} \propto \varphi^{0} \quad \text { or }\left\langle R^{2}\right\rangle \propto N \varphi^{0},
$$

in agreement with static scaling laws. ${ }^{2,14}$ The small $O\left(N^{-1}\right) \varphi^{-1}$ correction due to end chain site mutation vanishes in the infinite chain limit. Thus, a simple microscopic picture emerges from the chain length fluctuations caused by density fluctuations: As solvent concentration increases, solvent sites start appearing with (to first order) equal probability throughout the system, at the cost of a decrease in chain length. Mutation of a single mer into a solvent site accompanied by splitting of the chain (type IV) is the mechanism that dominates the $\left\langle R^{2}\right\rangle$ vs $\varphi$ dependence.

It is essential to realize that the use of the $\widetilde{\Xi}$ ensemble offers a quite different, but complementary, view to the usual description of chain shrinkage at fixed chain length $N$, as in the usual $\left[N n P T \mu^{*}\right]$ ensembles. ${ }^{33,35}$ In the $\widetilde{\Xi}$ ensemble transitions between states imply fluctuations in number average degree of polymerization $N$, whereas $N$ is strictly constant in an $\left[N n P T \mu^{*}\right]$ ensemble. ${ }^{33}$ From the $\widetilde{\Xi}$ point of view, as polymer volumetric fraction fluctuates away from $\varphi^{\mathrm{MRJ}}$, chains become shorter predominantly by "breakage" (type IV tran- sitions), in favor of an increase in $\varphi_{\text {sol }}$, so that the $\left\langle R^{2}\right\rangle$ $\propto N \varphi^{0}$ dependence results.

Furthermore an estimation of the cross-over concentration from concentrated to marginal can be made by realizing that the mechanism summarized in Eq. (24) must cease to be dominant when type IV transitions become increasingly rare due to the lower bound in chain length set by the length scale separation (or equivalently, chemical potential spectrum). Regardless of the details of the distribution this must happen at a solvent volumetric fraction $\varphi_{\text {sol }}=1-\varphi^{* \text {,conc }}$ where individual solvent sites cease to be entirely surrounded by chain sites. A reasonable first-order value for infinite chain length is

$$
\varphi_{\text {sol }}^{* \text {,onc }}=\frac{1}{n_{\text {coord }}} \varphi^{\mathrm{MRJ}} \simeq 0.046 \Rightarrow \varphi_{\infty}^{* \text {,onc }}=0.59,
$$

where $n_{\text {coord }}$ is the average coordination number of a site in a $\mathbf{R}_{j}$ configuration (at MRJ, $n_{\text {coord }}=14$ ). ${ }^{24}$ It is possible to obtain a correction to this estimate, by considering a solution of number average chain length $N$ at its cross-over concentration $\varphi^{*, \operatorname{conc}}(N)$. Consider a fluctuation $\delta N$ to a new, higher value $N^{\prime}=N+\delta N$. The occurrence of this fluctuation implies that type IV transitions, which had ceased to be dominant at $\varphi^{* \text {,conc }}(N)$, will regain statistical weight until the new, larger average $N^{\prime}$ at the corresponding $\varphi^{*, \text { conc }}\left(N^{\prime}\right)$ is reached. Since chains are, on average, larger in this fluctuation $\left(N^{\prime}>N\right)$, the number of states available in which type IV transitions are possible will necessarily also be larger, i.e., the fluctuation to a larger molecular weight $N^{\prime}$ carries the system in a region of configuration space where type IV can again take place. Hence, the system with larger molecular weight must find itself farther away from the cross-over concentration than the short-chain configurations. The implication is that Eq. (26) should be modified to

$$
\varphi^{*, \text { conc }}(N) \propto \varphi_{\infty}^{*, \text { conc }}\left(1+O\left(N^{-1}\right)\right) .
$$

While the numerical prefactor in the $O\left(N^{-1}\right)$ correction cannot be predicted, it must however be strictly positive, that is, the transition concentrated $\leftrightarrow$ marginal must take place at higher $\varphi$ (lower $\varphi_{\text {sol }}$ ) the shorter the chains are.

The above discussion is based on fluctuations away from $\varphi^{\mathrm{MRJ}}$, where transitions reduce $N$. Needless to say the reverse argumentation in which $N$ grows by mutation of solvent sites 


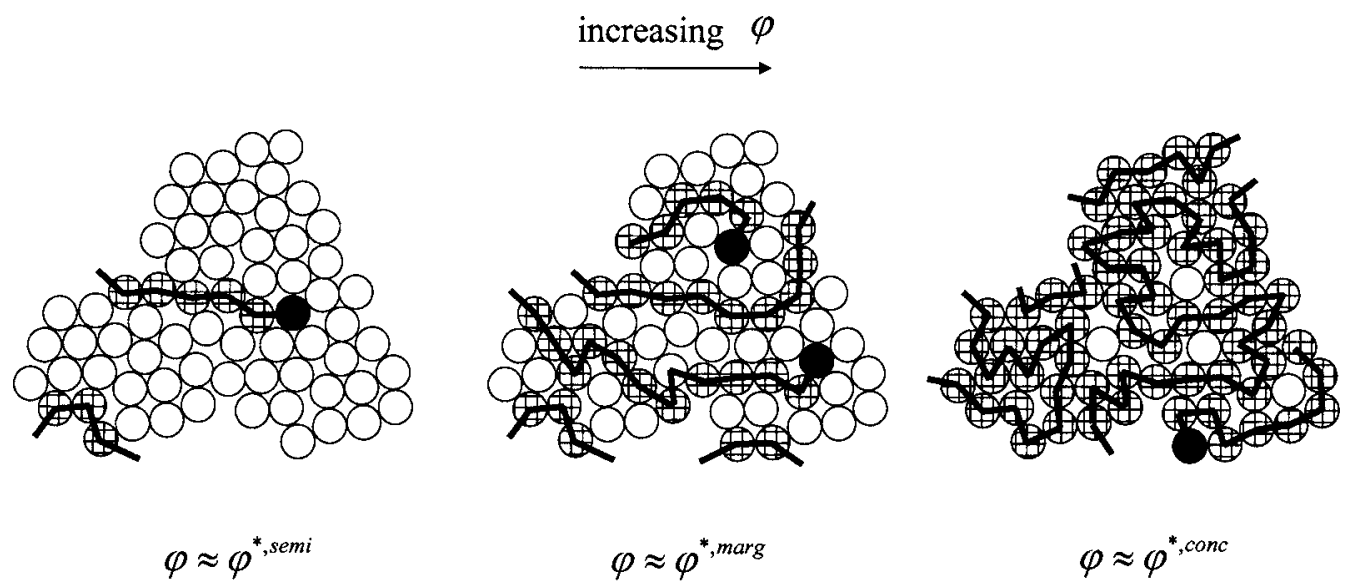

FIG. 4. Qualitative representation of conditions in semidilute, marginal, and concentrated regimes.

into chain sites, is equally valid since microscopic reversibility is taken into account in Eqs. (23) and (24).

\section{MARGINAL REGIME}

At $\varphi<\varphi^{* \text {,conc }}$ type III transitions (lateral accretion) become the dominant mechanism for density fluctuations so that the expected scaling will now be

$$
C_{N}=\frac{\left\langle R^{2}\right\rangle}{N b^{2}} \propto \varphi^{-1} \quad \text { or }\left\langle R^{2}\right\rangle \propto N \varphi^{-1} .
$$

In the constant- $N$ view, a chain undergoes collapse as $\varphi$ is increased. The decrease of $R^{2}$ at fixed $N$ results in $\left\langle R^{2}\right\rangle$ $\propto N \varphi^{-1}$. In the $\widetilde{\Xi}$ view, a chain grows by "side accretion" of solvent sites without changing its end-to-end distance: Solvent volume fraction is converted in polymer volume fraction at constant chain size, hence, the same $\left\langle R^{2}\right\rangle \propto N \varphi^{-1}$ results. From this perspective, one can grasp very intuitively how chain collapse can also be viewed as a "thickening" of the coil by accretion (identity change) of surrounding solvent sites at constant end-to-end distance. While equivalent to other semigrand ensembles in the thermodynamic limit, the advantage of $\widetilde{\Xi}$ is that volumetric fraction and size fluctuations are correlated quite transparently.

The volumetric fraction at the cross-over from marginal to semidilute can be estimated by considering that type III transitions cease to be dominant when alternative pathways involving more than one solvent site become highly probable. This can be estimated to happen at a $\varphi^{*, \text { marg }}$ at which individual chain molecules are separated from each other on average by a solvent site. ${ }^{54}$ From a geometric point of view, at $\varphi^{*, \text { conc }}>\varphi>\varphi^{* \text {,marg }}$, it is highly probable that solvent sites will be in a (double or multiple) tangency position to chain molecules, and therefore in a configuration favorable for being incorporated into an existing chain in a type III transition. An estimate of $\varphi^{* \text {,marg }}$ can again be based on a simple geometrical condition: Packing tubelike, fractal or not, objects in three-dimensional space so that they are separated, on average, by the size of a solvent site suggests

$$
\varphi_{\infty}^{*, \operatorname{marg}} \simeq \frac{1}{2} \varphi^{\mathrm{MRJ}}=0.32 .
$$

An argument entirely similar to the one presented above for the concentrated regime leads to a following correction:

$$
\varphi^{*, \operatorname{marg}}(N) \propto \varphi_{\infty}^{*, \operatorname{marg}}\left(1+O\left(N^{-1}\right)\right) .
$$

In analogous fashion, the numerical prefactor in the $O\left(N^{-1}\right)$ correction must be strictly positive, and the transition marginal $\leftrightarrow$ semidilute must take place at higher $\varphi$ for shorter chains. Both corrections to the cross-over concentrations also imply that the transitions at $\varphi^{*, \operatorname{marg}}(N)$ and $\varphi^{*, \operatorname{conc}}(N)$ become more diffuse for low $N$.

The two estimates Eqs. (26) and (29) are satisfyingly dual in the sense that they represent limiting cases of a solvent site being surrounded by chain sites [Eq. (26)] and a chain site surrounded by solvent sites [Eq. (29)] (see Fig. 4). It is interesting to note the correspondence of Eqs. (26) and (29) with standard thermodynamic arguments: $\varphi_{\infty}^{* \text {,marg }}$ is defined to lie at the volumetric fraction at which quadratic terms in the Flory-Huggins expansion become dominant. ${ }^{13}$ The prevalence of the quadratic term corresponds to the onset of chain-chain binary interactions, which in microscopic terms starts when chains are separated on average by one solvent site. Similarly, $\varphi_{\infty}^{*, \text { conc }}$ is associated with "higher order terms becoming dominant," 13 which is a roundabout way of stating that binary solvent-solvent interactions cease to be important. The $\widetilde{\Xi}$ ensemble offers a more symmetric, microscopic point of view: $\varphi_{\infty}^{* \text {,conc }}$ is found where binary solvent-solvent interactions acquire statistical importance as volume fraction decreases. The basic geometrical difference between the dimensionalities of solvent and polymer is ultimately responsible for there being two cross-over transitions at high concentrations: A chain molecule surrounded by solvent molecules at $\varphi_{\infty}^{* \text {,marg }}$ and a solvent molecule surrounded by chain molecules at $\varphi_{\infty}^{*, \text { conc }}$. As size and shape differences between solvent and polymer decreases, so must the sharpness of the transitions at $\varphi_{\infty}^{* \text {,marg }}$ and $\varphi_{\infty}^{* \text {,conc }}$ decrease as well.

Several simplifications used in the previous sections depended on the simplicity of the hard-sphere interaction potential and on the exact surjection between $\widetilde{\Xi}$ and $S$. To what extent then do the predicted static scaling laws and cross- 


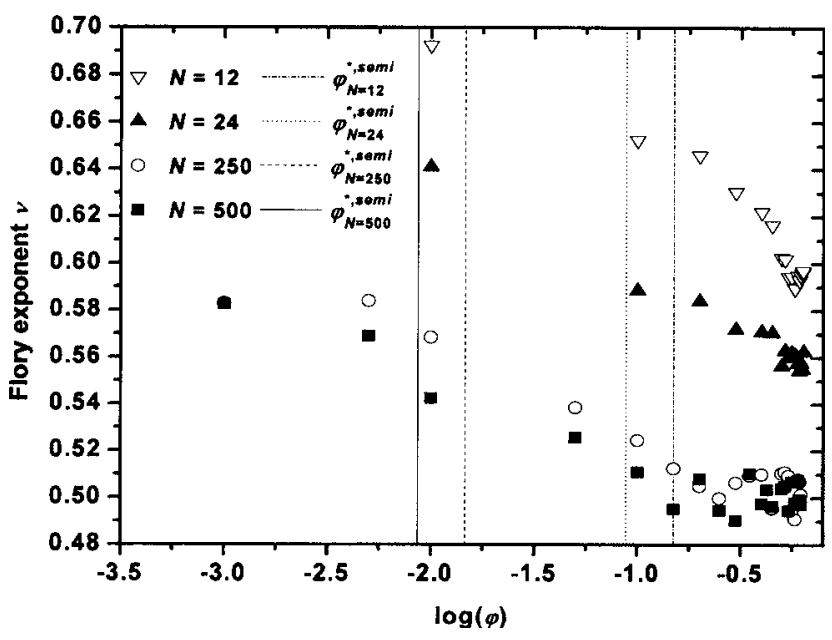

FIG. 5. Flory scaling exponent as a function of logarithm of packing density $\varphi$ from MC simulations. Lines mark $\varphi^{* \text {,semi }}$ (transition from dilute to semidilute) from Ref. 2, for different chain lengths.

over volumetric fractions reflect universal behavior? On one hand, the surjection between $\widetilde{\Xi}$ and $S$ allows the formalization of some arguments, but is not essential. MRJ states are multicontact, mechanically isostatic packings, which implies a large number of tangencies between individual spherical sites, ${ }^{56}$ as well as extremely high chain flexibility. In real polymer solutions, chain flexibility and average coordination number between solvent and chain sites will be lower. Both will depend on molecular geometry and on chemical details. However, these will not alter the fundamental difference between lateral and end accretion mechanisms in correlated density-chain length fluctuations. Hence, Eqs. (25), (27), (28), and (30) should be valid for general flexible homopolymer-solvent systems. Furthermore, the chemical similarity between solvent and chain sites implicit in Eq. (15) cannot hold exactly in general. Specific solvent-mer interactions may alter prefactors, narrow or even suppress the marginal regime in extreme cases. While these effects will necessarily be reflected in the numerical values of the characteristic ratio, they should not modify scaling as in Eqs. (25), (27), (28), and (30). Finally, the very natural assumption of solvent-polymer length scale separation is clearly essential: None of the predictions made above can possibly hold if the lengths of polymer and solvent molecules do not differ significantly.

\section{COMPARISONS WITH MC RESULTS}

The results of a large set of MC simulations at different densities $^{24,25}$ are summarized in Figs. 5 and 6 for systems of freely jointed chains of hard spheres characterized by average molecular lengths of $N=12,24,250$, and 500 . Thanks to the efficient MC scheme these simulations explore the entire density range $0<\varphi<\varphi^{\mathrm{MRJ}}$. Despite their simplicity, hardsphere chains undoubtedly display four clearly distinct scaling regimes of universal scaling for $\left\langle R^{2}\right\rangle$ on $N$ and $\varphi$, from dilute to melt. The results shown in Figs. 6 and 7 leave little room for doubt about the observability of marginal and concentrated regimes. After the dilute and semidilute regimes, where previously observed ${ }^{18}$ scalings are confirmed, the mar-

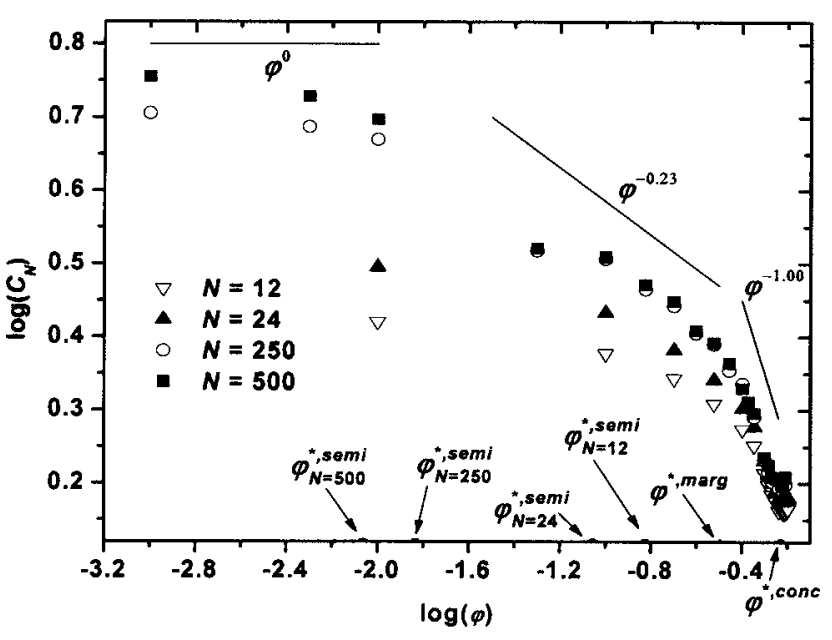

FIG. 6. Log-log plot of characteristic ratio $C_{N}$ as a function of density $\varphi$ for several chain lengths. Straight lines with slopes given by the expected scaling exponents are drawn as an aide to the eye (except in the concentrated regime, where $\varphi^{0}$ scaling is predicted, see Fig. 7). Arrows mark $\varphi^{* \text {,semi }}$ (transition from dilute to semidilute) from Ref. 2, and also cross-over densities $\varphi^{* \text {,marg }}$ and $\varphi^{* \text {,conc }}$ predicted by Eqs. (29) and (26) for $N \rightarrow \infty$, at which semidilute scaling $\left\langle R^{2}\right\rangle \sim \varphi^{-0.23}$ changes to marginal scaling $\left\langle R^{2}\right\rangle \sim \varphi^{-1}$, and marginal changes to concentrated scaling $\left\langle R^{2}\right\rangle \sim \varphi^{0}$, respectively.

ginal regime appears at approximately $\varphi^{*, \text { marg }} \approx 0.3$, characterized by a scaling law $\left\langle R^{2}\right\rangle \sim N \varphi^{-1}$. This transition is quite sharp. Schaefer's claim that chain flexibility suppresses the marginal regime ${ }^{14}$ is not supported by these results. Above $\varphi^{* \text {,conc }} \approx 0.58$ a concentrated regime behavior characterized by $\left\langle R^{2}\right\rangle \sim N \varphi^{0}$ is shown in the linear-log plot of Fig. 7. As Fig. 5 shows, Flory's exponent for long chains is identical to 0.5 within very small statistical error bars throughout the marginal and concentrated regimes.

The estimates for the cross-over volumetric fractions [Eqs. (26) and (29)] are unreasonably accurate, certainly due to large chain flexibility, and to the high coordination number in packings of hard-sphere chains. Cross-over volumetric fractions for more realistic systems will undoubtedly depart

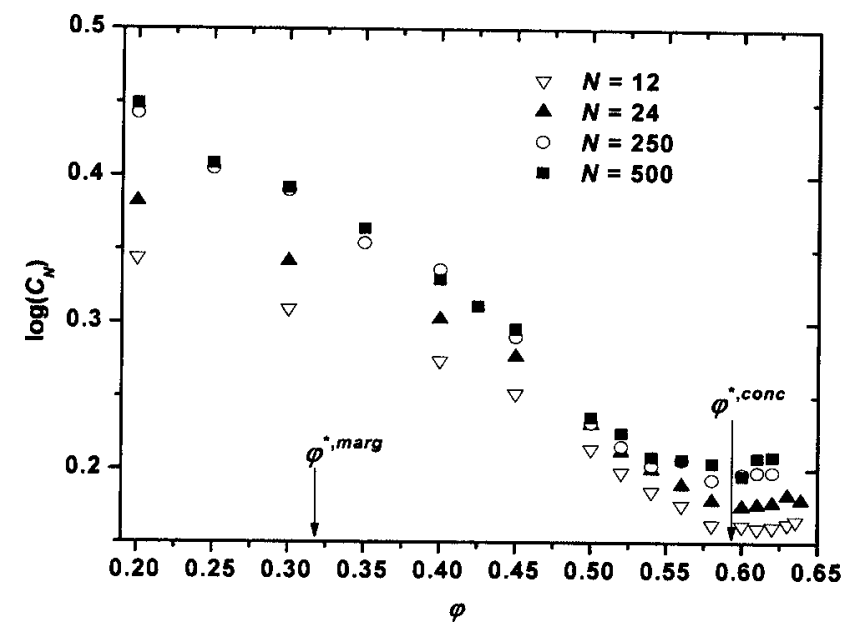

FIG. 7. Logarithm of the characteristic ratio $\log \left(C_{N}\right)$ as a function of packing density $\varphi$ as obtained from MC simulations focusing on the marginal and concentrated regimes. Predicted $\varphi^{0}$ scaling is clearly observable above $\varphi^{*}$,conc . Also shown are the theoretically predicted cross-over densities (for $N \rightarrow \infty)$ for the transitions (i) semidilute $\leftrightarrow$ marginal $\left(\varphi^{*}\right.$ marg $)$ and (ii) marginal $\leftrightarrow$ concentrated $\left(\varphi^{*, \text { conc }}\right)$. 
from these values. The chain length corrections [Eqs. (27) and (30)] are qualitatively correct: Concentrated and marginal cross-overs shift to lower volumetric fractions, and the corresponding regimes widen as chain length increases. It is not possible to reliably verify the exponent in the correction terms in Eqs. (27) and (30), although it seems not to be too different from the predicted -1 .

\section{CONCLUSIONS}

The use of a semigrand ensemble has enable predictions for universal scaling of chain dimensions on polymer volumetric fraction and of cross-over to marginal and to concentrated regimes. These predictions are based on graph theoretical arguments that identify those microsocopic configurational transitions that contribute to density-chain length fluctuations. Both cross-over volumetric fractions are found to be chain length independent to first order, although weaker $N^{-1}$ corrections are also predicted. These predictions are confirmed by an extensive set of MC calculations covering the full $\varphi$ range. Thus, it seems that the hard-sphere chain model contains all the essential physics that determine the statics of long chain molecules both in solution and in the melt. Hence it has continued value as foundation for perturbation theory and as statistical mechanics workbench.

\section{ACKNOWLEDGMENTS}

We are deeply grateful to the EC for financial support of the present project through Contract Nos. G5RD-CT-200200720 (PMILS) and NMP3-CT-2005-016375 (NSF-EC collaboration project MNIBS) and by CICYT through Contract No. MAT2005-25569-E. All reported simulations were conducted on the "Magerit" supercomputer of CeSViMa (UPM, Spain).

${ }^{1}$ P. J. Flory, Statistical Mechanics of Chain Molecules (Hanser Verlag, Munich, 1989).

${ }^{2}$ P. G. de Gennes, Scaling Concepts in Polymer Physics (Cornell University Press, Ithaca, 1980).

${ }^{3}$ M. Doi and S. F. Edwards, The Theory of Polymer Dynamics (Clarendon, Oxford, 1989).

${ }^{4}$ M. Daoud, J. P. Cotton, B. Farnoux, G. Jannink, G. Sarma, H. Benoit, R. Duplessix, C. Picot, and P. G. de Gennes, Macromolecules 8, 804 (1975).

${ }^{5}$ J. P. Cotton, M. Nierlich, F. Boué, M. Daoud, B. Farnoux, G. Jannink, R. Duplessix, and C. Picot, J. Chem. Phys. 65, 1101 (1976).

${ }^{6}$ B. Farnoux, F. Boue, J. P. Cotton, M. Daoud, G. Jannink, M. Nierlich, and P. G. de Gennes, J. Phys. (France) 39, 77 (1978).

${ }^{7}$ We follow the widespread convention of using $N$ (no subindex) for a generic chain length in a scaling law. It should not cause confusion with the number of chains $N_{i}$ of a particular length $i$.

${ }^{8}$ W. Paul, K. Binder, D. W. Heermann, and K. Kremer, J. Phys. II 1, 37 (1991).

${ }^{9}$ P. Cifra and T. Bleha, Polymer 34, 4924 (1993).

${ }^{10}$ A. Yethiraj and C. K. Hall, J. Chem. Phys. 96, 797 (1991); A. Yethiraj and R. Dickman, ibid. 97, 4468 (1992).

${ }^{11}$ A. J. Haslam, G. Jackson, and T. C. B. McLeish, J. Chem. Phys. 111, 416 (1999).

${ }^{12}$ R. G. Larson, J. Polym. Sci., Part B: Polym. Phys. 45, 3240 (2007).

${ }^{13}$ G. J. Fleer, M. A. Cohen Stuart, J. M. H. M. Scheutjens, T. Cosgrove, and B. Vincent, Polymers at Interfaces (Chapman and Hall, London, 1993).

${ }^{14}$ D. W. Schaefer, Polymer 25, 387 (1984).

${ }^{15}$ S. F. Edwards, Proc. Phys. Soc. London 88, 265 (1966).

${ }^{16}$ R. W. Richards, A. Maconnachie, and G. Allen, Polymer 19, 266 (1978); 22, 147 (1981); 22, 153 (1981); 22, 158 (1981).
${ }^{17}$ B. Hammouda, Adv. Polym. Sci. 106, 87 (1993).

${ }^{18}$ G. D. Wignall and Y. B. Melnichenko, Rep. Prog. Phys. 68, 1761 (2005).

${ }^{19}$ J. P. Wittmer, P. Beckrich, F. Crevel, C. C. Huang, A. Cavallo, T. Kreer, and H. Meyer, Comput. Phys. Commun. 177, 146 (2007); J. P. Wittmer, P. Beckrich, H. Meyer, A. Cavallo, A. Johner, and J. Baschnagel, Phys. Rev. E 76, 011803 (2007); J. P. Wittmer, P. Beckrich, A. Johner, A. N. Semenov, S. P. Obukhov, H. Meyer, and J. Baschnagel, EPL 77, 56003 (2007).

${ }^{20}$ P. Beckrich, A. Johner, A. N. Semenov, S. P. Obukhov, H. Benoit, and J. P. Wittmer, Macromolecules 40, 3805 (2007).

${ }^{21}$ M. Kröger, Comput. Phys. Commun. 118, 278 (1999).

${ }^{22}$ M. Kröger, M. Müller, and J. Nievergelt, Comput. Model. Eng. Sci. 4, 559 (2003).

${ }^{23}$ M. Rosche, R. G. Winkler, P. Reineker, and M. Schulz, J. Chem. Phys. 112, 3051 (2000)

${ }^{24}$ N. C. Karayiannis and M. Laso, Phys. Rev. Lett. 100, 050602 (2008).

${ }^{25}$ N. C. Karayiannis and M. Laso, Macromolecules 41, 1537 (2008).

${ }^{26}$ S. Torquato, T. M. Truskett, and P. G. Debenedetti, Phys. Rev. Lett. 84, 2064 (2000).

${ }^{27}$ A. Donev, F. H. Stillinger, and S. Torquato, Phys. Rev. Lett. 95, 090604 (2005).

${ }^{28}$ Z. H. Stachurski, Phys. Rev. Lett. 90, 155502 (2003).

${ }^{29}$ Solutions in supercritical solvents, where incompressibility is not fulfilled, are thus excluded from the present discussion.

${ }^{30}$ J. G. Briano and E. D. Glandt, J. Chem. Phys. 80, 3336 (1984).

${ }^{31}$ D. A. Kofke and E. D. Glandt, Mol. Phys. 64, 1105 (1988).

${ }^{32}$ P. G. Bolhuis and D. A. Kofke, Phys. Rev. E 54, 634 (1996).

${ }^{33}$ P. V. K. Pant and D. N. Theodorou, Macromolecules 28, 7224 (1995).

${ }^{34}$ F. A. Escobedo and J. J. de Pablo, Macromolecules 32, 900 (1999).

${ }^{35}$ F. A. Escobedo, J. Chem. Phys. 115, 5642 (2001).

${ }^{36}$ M. Doxastakis, V. G. Mavrantzas, and D. N. Theodorou, J. Chem. Phys. 115, 11352 (2001).

${ }^{37}$ F. L. Colhoun, R. C. Armstrong, and G. C. Rutledge, Macromolecules 35, 6032 (2002).

${ }^{38}$ F. A. Escobedo, J. Chem. Phys. 118, 10262 (2003).

${ }^{39}$ T. Kristof and I. Szalai, Phys. Rev. E 72, 041105 (2005).

${ }^{40}$ J. de Joannis, Y. Jiang, F. Yin, and J. T. Kindt, J. Phys. Chem. B 110, 25875 (2006).

${ }^{41}$ N. C. Karayiannis, V. G. Mavrantzas, and D. N. Theodorou, Phys. Rev. Lett. 88, 105503 (2002); N. C. Karayiannis, A. E. Giannousaki, V. G. Mavrantzas, and D. N. Theodorou, J. Chem. Phys. 117, 5465 (2002).

${ }^{42}$ N. C. Karayiannis, A. E. Giannousaki, and V. G. Mavrantzas, J. Chem. Phys. 118, 2451 (2003)

${ }^{43}$ B. Widom, J. Chem. Phys. 39, 2805 (1963).

${ }^{44}$ D. A. McQuarrie, Statistical Mechanics (Harper \& Row, New York, 1976).

${ }^{45}$ There is a subtle point regarding the volumetric fraction: $\varphi$ cannot be specified directly, but results from the imposed spectrum of chemical potentials. The prescription [Eq. (12)] implies that $\left.\left\langle N_{1}\right\rangle\right\rangle\left\langle N_{i}\right\rangle, \forall i \neq 1$, which seems to preclude the possibility of exploring regimes where $\varphi_{\text {sol }} \rightarrow 0, \varphi \rightarrow \varphi^{\mathrm{MRJ}}$. In fact, $\varphi$ can be controlled, for a sufficiently large, fixed $N_{\text {sites }}$, by varying $l_{\min }$ while keeping $l_{\min } \gg 1$, or if we fix $l_{\min } \gg 1$, by considering increasingly larger ensemble sizes. In both cases, although $\left\langle N_{1}\right\rangle>\left\langle N_{i}\right\rangle, \forall i \neq 1$, it is possible to achieve $\left\langle N_{1}\right\rangle \ll \Sigma_{i}\left\langle N_{i}\right\rangle, \forall i \neq 1$ and thus let $\varphi \rightarrow \varphi^{\mathrm{MRJ}}$.

${ }^{46}$ S. K. Kumar, J. Chem. Phys. 96, 1490 (1992).

${ }^{47}$ P. M. Chaikin and T. C. Lubensky, Principles of Condensed Matter Physics (Cambridge University Press, Cambridge, 2000).

${ }^{48}$ J. Pach and P. K. Agarwal, Combinatorial Geometry (Wiley, New York, 1995).

${ }^{49}$ J. Sun, S. Boyd, L. Xiao, and P. Diaconis, SIAM Rev. 48, 681 (2006).

${ }^{50}$ A. Pothen, H. Simon, and K. Liou, SIAM J. Matrix Anal. Appl. 11, 430 (1990).

${ }^{51}$ P. Brémaud, Markov Chains: Gibbs Fields, Monte Carlo Simulation, and Queues (Springer, New York, 1999).

${ }^{52}$ M. Jerrum and A. Sinclair, SIAM J. Comput. 18, 1149 (1989).

${ }^{53}$ R. A. Ahuja, T. L. Magnanti, and J. B. Orlin, Network Flows: Theory, Algorithms and Application (Prentice Hall, New Jersey, 1993).

${ }^{54}$ This is quite different from (incorrect) argumentations based on a polymer chain being "coated" in a solvent layer at the $\theta$ state, as pointed out in Ref. 55.

${ }^{55}$ S. T. Milner, Macromolecules 38, 4929 (2005).

${ }^{56}$ A. Donev, S. Torquato, and F. H. Stillinger, Phys. Rev. E 71, 011105 (2005). 